\title{
FORWARD I: a Phase III study of mirvetuximab soravtansine versus chemotherapy in platinum-resistant ovarian cancer
}

\author{
Kathleen N Moore ${ }^{*}, 1$, Ignace Vergote ${ }^{2}$, Ana Oaknin ${ }^{3}$, Nicoletta Colombo ${ }^{4}$, Susana \\ Banerjee $^{5}$, Amit Oza ${ }^{6}$, Patricia Pautier ${ }^{7}$, Karim Malek $^{8}$ \& Michael J Birrer ${ }^{9}$ \\ ${ }^{1}$ Department of Obstetrics \& Gynecology, Stephenson Oklahoma Cancer Center at the University of Oklahoma Health Sciences \\ Center, Oklahoma City, OK 73104, USA \\ ${ }^{2}$ Gynaecological Oncology, Leuven Cancer Institute, Leuven 3000, Belgium \\ ${ }^{3}$ Medical Oncology Department, Vall D'Hebron Institute of Oncology (VHIO), Barcelona 08035, Spain \\ ${ }^{4}$ Gynecologic Oncology, The European Institute of Oncology, Milan 20141, Italy \\ ${ }^{5}$ Gynaecology Unit, Royal Marsden Hospital, London, SW3 6JJ, UK \\ ${ }^{6}$ Division of Medical Oncology \& Hematology, Princess Margaret Cancer Centre, Toronto M5G 2M9, Canada \\ ${ }^{7}$ Department of Adult Medicine, Gustave Roussy Cancer Campus, Villejuif 94800, France \\ ${ }^{8}$ Clinical Development, ImmunoGen, Inc., Waltham, MA 02451, USA \\ ${ }^{9}$ Division of Hematology-Oncology, University of Alabama at Birmingham Comprehensive Cancer Center, Birmingham, AL 35294 \\ USA \\ *Author for correspondence: Tel.: +1 405271 8707; kathleen-moore@ouhsc.edu
}

Mirvetuximab soravtansine, an antibody-drug conjugate that binds with high affinity to folate receptor$\alpha$ to provide tumor-directed delivery of the potent microtubule-disrupting agent DM4, has emerged as a promising investigational agent for the treatment of ovarian cancer, particularly in the setting of platinum-resistant disease. Here we describe the rationale and design of FORWARD I (NCT02631876), the first randomized, multicenter Phase III study to compare the safety and efficacy of mirvetuximab soravtansine versus investigator's choice of chemotherapy in women with folate receptor- $\alpha$-positive, platinumresistant epithelial ovarian, primary peritoneal or fallopian tube cancer. Patients will be randomized in a 2:1 ratio. The primary end point is progression-free survival, and key secondary objectives include comparison of overall response rates, overall survival and duration of response.

First draft submitted: 20 November 2017; Accepted for publication: 25 January 2018; Published online: 9 February 2018

Keywords: antibody-drug conjugate • folate receptor- $\alpha \bullet$ mirvetuximab soravtansine $\bullet$ ovarian cancer $\bullet$ Phase III trial • targeted therapy

Ovarian cancer is a leading cause of gynecologic cancer mortality worldwide, responsible for over 150,000 deaths each year [1]. Family history and the presence of penetrant mutations in genes such as BRCA1 and BRCA2 are significant risk factors for the development of ovarian cancer; epidemiological research has also identified a variety of hormonal and reproductive factors that can either increase (e.g., older age at menopause, hormone replacement therapy) or decrease (e.g., parity, lactation, oral contraceptive use) the likelihood of disease (reviewed in [2]). Epithelial ovarian cancer (EOC) accounts for approximately $95 \%$ of ovarian cancer malignancies [3,4]. EOC is highly chemosensitive, with the current standard-of-care treatment for newly diagnosed cases involving debulking surgery and adjuvant platinum- and taxane-based combination chemotherapy. Most individuals achieve remission with front-line therapy; unfortunately up to $80 \%$ of patients will relapse during or after treatment with eventual drug-resistant disease [5]. Disease recurring within 6 months of platinum-based chemotherapy is classified as platinum-resistant, whereas disease recurring $>6$ months after therapy is considered platinum sensitive. Patients with platinum-sensitive disease have a high likelihood of responding to additional platinum-based therapy; however, almost all will eventually develop acquired (secondary) platinum resistance [5,6]. Patients with platinum-resistant

Future Medicine 
ovarian cancer typically receive single-agent chemotherapy at relapse. Outcomes for these patients remain poor, with low response rates to further chemotherapy (15-20\%), progression-free survival (PFS) of 3-4 months [7-9], and a median overall survival rate of less than a year [5]. In addition, this treatment approach is associated with additional, cumulative toxicities and limited tolerability for patients. It has been suggested that natural compounds, such as phytochemicals, may alleviate chemotherapy-induced side effects and therefore may be useful as adjuvants to standard treatment [10]; however, this field remains in its infancy. These measures underscore a critical need for innovative and effective therapeutic strategies for the management of advanced ovarian cancer.

Due to an increased understanding of the biological and genomic complexity of EOC, recent progress has been made in the clinical application of molecularly targeted strategies designed to shift treatment away from broadbased cytotoxic use toward more tailored therapeutic interventions [11,12]. Approval of the angiogenesis inhibitor bevacizumab and the poly (ADP-ribose) polymerase inhibitors olaparib, rucaparib and niraparib [13,14], in both treatment and maintenance settings, highlights the degree to which targeted agents are presently impacting the treatment landscape for patients with advanced ovarian cancer. Another candidate molecule of considerable interest for the development of novel therapeutics is the $\mathrm{FR} \alpha$, a glycosylphosphatidylinositol-anchored cell surface protein encoded by the FOLR1 gene [15]. FR $\alpha$ mediates the active transport of folate, a B vitamin essential for cellular metabolism as well as DNA synthesis, methylation and repair [16], into cells via the process of receptor-mediated endocytosis. In contrast to a highly restricted distribution pattern in normal tissues, aberrant overexpression of FR $\alpha$ is characteristic of a variety of epithelial tumors, including ovarian cancer [15]. Indeed, independent studies have reported $80-96 \%$ of ovarian tumors having constitutive expression of $F R \alpha$, which is absent in the normal ovarian epithelium [15,17-19], and elevated receptor expression has been proposed to be a negative prognostic factor in terms of chemotherapeutic response in this malignancy [20]. Previously, a number of experimental FR $\alpha$-targeting agents designed to exploit this differential distribution pattern as an avenue of therapeutic intervention in EOC have undergone clinical evaluation [21]. These included the humanized anti-FR $\alpha$ monoclonal antibody farletuzumab and small molecule folate-cytotoxic agent conjugate vintafolide [22,23]. Both agents were well tolerated in human trials; however, their clinical development was hampered due to a lack of meaningful single-agent activity in ovarian cancer patients and only modest efficacy in pivotal trials $[24,25]$.

The inherent capacity of FR $\alpha$ to internalize large molecules makes this receptor well suited for antibodydrug conjugate (ADC)-based therapeutic approaches. ADCs are complex engineered molecules comprised of a monoclonal antibody, directed toward tumor-associated antigens, conjugated to a potent cytotoxic agent ('payload') via stable linkers. Accordingly, ADCs couple the targeting and pharmacokinetic features of the antibody moiety with the additional cancer-killing impact of the payload [26]. This method of site-selective drug delivery affords a mechanism to reduce off-target toxicities in patients by limiting the exposure of normal tissues to the payload [27]. Currently there are four ADCs approved for cancer treatment: brentuximab vedotin (a conjugate of an antiCD30 antibody with monomethyl auristatin E), ado-trastuzumab emtansine (T-DM1, a conjugate of the antiHER2 antibody trastuzumab with the maytansinoid compound DM1), inotuzumab ozogamicin and gemtuzumab ozogamicin (conjugates of CD22- and CD33-targeting antibodies, respectively, with calicheamicin) [28]. In addition, there are over 60 more presently under evaluation in a variety of hematological and solid tumors [29,30].

\section{Mirvetuximab soravtansine}

Mirvetuximab soravtansine (formerly IMGN853) is an ADC consisting of a humanized FR $\alpha$-binding monoclonal antibody coupled, via a cleavable disulfide linker, to the maytansinoid effector molecule DM4 [31]. Each antibody molecule is conjugated with an average of three to four molecules of DM4, which acts as a potent antimitotic agent through its ability to suppress microtubule dynamics [32,33]. Following high affinity binding to surfaceexpressed FR $\alpha$, functional activation of mirvetuximab soravtansine requires internalization and catabolism in order to release the cytotoxic payload. A model for mirvetuximab soravtansine processing and mechanism of action is presented in Figure 1. Internalization occurs via antigen-mediated endocytosis, following which the conjugate molecule is delivered to lysosomes by vesicular trafficking, and then degraded to release lysine-Ne-sulfo-SPDBDM4. This catabolite undergoes further intracellular reduction and S-methylation, leading to the formation of the hydrophobic maytansinoid derivatives, DM4 and S-methyl-DM4. All three metabolites exert potent antimitotic activity, via inhibition of tubulin polymerization and microtubule assembly, resulting in cell death. Of note, the last two hydrophobic derivatives are able to diffuse from antigen-positive tumor cells into neighboring cells and kill them in an antigen-independent manner. This effect, known as 'bystander' killing [34], is beneficial in vivo where $\mathrm{FR} \alpha$ expression on tumor cells is heterogeneous, and/or where tumor penetration of the ADC molecule may be 


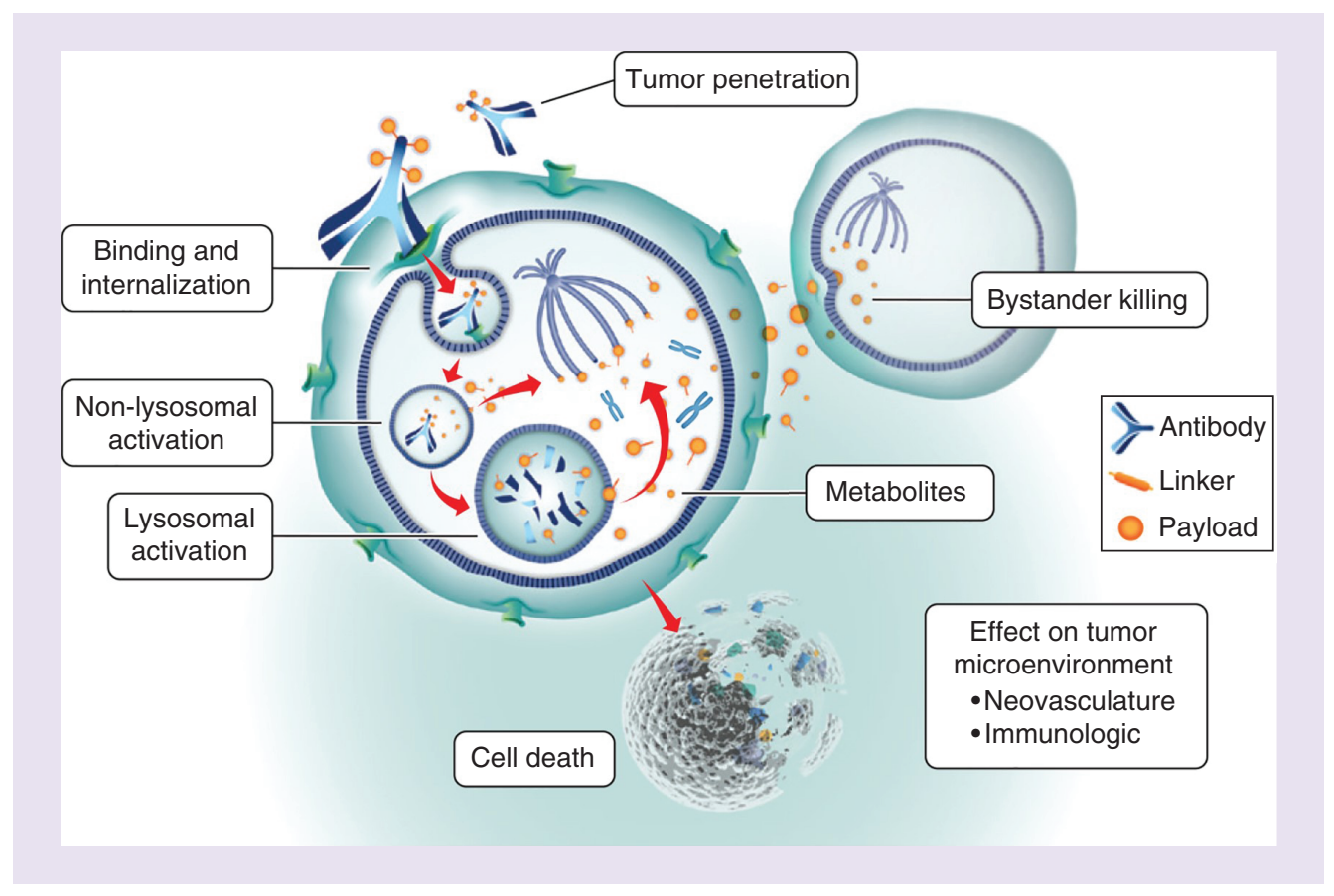

Figure 1. Mirvetuximab soravtansine mechanism of action. Mirvetuximab soravtansine binds with high affinity to folate receptor- $\alpha$ expressed on the tumor cell surface, prompting internalization of the antibody-drug conjugate/receptor complex via antigen-mediated endocytosis. Lysosomal processing releases active DM4 catabolites - these maytansinoid derivatives inhibit tubulin polymerization and microtubule assembly, inducing potent antimitotic effects that result in cell-cycle arrest and apoptosis. The active metabolites may also diffuse into neighboring cells and induce further cell death (termed 'bystander killing').

Reproduced with permission from [30].

limited. Diffused cytotoxic metabolites may additionally exert effects on cells of the tumor microenvironment or neovasculature as a component of their antitumor activity [30].

The preclinical characterization of mirvetuximab soravtansine demonstrated that it reduced viability in FR $\alpha$ positive tumor cells in vitro with low nanomolar potency. Importantly, the level of surface receptor expression was identified as a key determinant of sensitivity, with those cells expressing higher levels of FR $\alpha$ being more sensitive to the cytotoxic effects of mirvetuximab soravtansine exposure [35]. Moreover, mirvetuximab soravtansine exhibited robust single-agent antitumor activity in vivo (at doses well below its maximum-tolerated dose) in multiple cell line and patient-derived xenograft models of ovarian and other FR $\alpha$-positive cancers [35]. Preclinical activity of this $\mathrm{ADC}$ in combination with standard-of-care therapeutics used in the management of ovarian cancer has also been reported [36] and those data provided the framework for an ongoing combination Phase Ib trial in patients with advanced ovarian cancer (FORWARD II).

\section{The FORWARD I study}

Here we describe the design and rationale for the FORWARD I study, an open-label, randomized Phase III trial to evaluate the safety and efficacy of mirvetuximab soravtansine versus investigator's choice (IC) chemotherapy in women with FR $\alpha$-positive, platinum-resistant EOC, primary peritoneal cancer or fallopian tube cancer. The study is funded by ImmunoGen, Inc., Waltham, MA, USA.

\section{Background \& rationale}

The first-in-human, Phase I study of single-agent mirvetuximab soravtansine in patients with EOC and other FR $\alpha$ positive solid tumors established the recommended Phase II dose (RP2D) as $6.0 \mathrm{mg} / \mathrm{kg}$, using adjusted ideal body weight (AIBW), administered once every 3 weeks [37]. The use of AIBW to calculate mirvetuximab soravtansine dosing was shown to reduce intracohort variability in pharmacokinetic exposure metrics. Mirvetuximab soravtansine was well tolerated as monotherapy, with the principal treatment-related toxicities seen being diarrhea, fatigue and 
nausea - the majority of cases were mild (grade 1 or 2) and readily managed without requiring discontinuation of therapy. Reversible ocular adverse events (AEs), primarily blurred vision and/or corneal keratopathy, were considered AEs of interest; these effects were considered off target due to a lack of FR $\alpha$ expression in the eye. In this regard, similar ocular AEs have been reported for a variety of ADCs that target different antigens and employ other tubulin-interacting payloads [38]. Early recognition prompted the implementation of daily lubricating eye drop use, alongside other symptomatic management procedures (e.g., avoidance of contact lenses, warm compress use, sunglasses in daylight, etc.), which subsequently decreased both the incidence and grade of visual disturbances in patients on study. In terms of antitumor activity, the strongest signals of clinical benefit to emerge during the dose escalation stage were in patients with EOC [37]. On the basis of this finding, additional expansion cohorts were opened as part of the same trial in individuals with advanced EOC, primary peritoneal or fallopian tube cancers. One characterized receptor expression in archival and fresh biopsy samples from a heterogeneous ovarian cancer population [39]; another evaluated safety and clinical activity in patients with platinum-resistant disease [40]; and a third investigated primary prophylactic use of corticosteroid eye drops while undergoing mirvetuximab soravtansine therapy. In all of these heavily pretreated patient populations, mirvetuximab soravtansine demonstrated encouraging signs of clinical activity that included confirmed objective tumor responses.

Two key observations to emerge from these expansion studies were instrumental in defining the target population for FORWARD I. First, a putative relationship between higher FR $\alpha$ expression and response to treatment was identified as part of the biopsy expansion cohort study [39]. An improved overall response rate (ORR) and extended PFS seen in individuals with the highest tumor FR $\alpha$ levels underscore the importance of incorporating patient stratification, based on receptor expression, into clinical trial design. Second, results from the defined platinumresistant EOC cohort suggested that the response rate correlated with the number of prior therapies [40]. In this cohort of 46 patients, the confirmed ORR was $26 \%$ and median PFS was 4.8 months. Subgroup analyses based on the number of previous therapies, however, demonstrated an ORR of $39 \%$ and a median PFS of 6.7 months in the subset of individuals enrolled with one to three priors (compared with 13\% and 3.9 months, respectively, for patients with four or more prior lines). Based on these considerations, FORWARD I is enrolling platinum-resistant patients who have received one to three prior therapies and who meet the FR $\alpha$ eligibility criterion of $\geq 50 \%$ tumor staining at $\geq 2+$ intensity (i.e., 'medium' to 'high' receptor expression). Robust support for this strategy was provided by retrospective pooled analysis across the entire Phase I trial [41]. Of a total of 113 ovarian cancer patients treated as part of the overall study, 36 platinum-resistant individuals were considered the 'FORWARD I-eligible' subset, meeting the criteria listed above. Of particular note, the efficacy measures in this group (confirmed ORR of $47 \%$ and median PFS 6.7 months) are superior to outcomes typically seen with established single-agent chemotherapy in the context of platinum-resistant disease.

With respect to the comparator chemotherapy arm of the study, the three most commonly used drugs in the setting of platinum resistance - paclitaxel, pegylated liposomal doxorubicin (PLD) and topotecan [7] - are being evaluated. Modified doses and administration schedules typically used in this setting are planned since the ability of patients to tolerate the bone marrow suppressive effects of cytotoxic chemotherapy are less than that of those receiving front-line therapy. The approved schedule of paclitaxel every 3 weeks for second-line treatment of ovarian cancer is associated with a high rate of myelosuppression, particularly neutropenia and peripheral neuropathy [42]. Therefore the antitumor activity and tolerability of an $80 \mathrm{mg} / \mathrm{m}^{2}$ weekly schedule, which has been reported to be associated with similar efficacy and an improved toxicity profile in several Phase II and III studies [43-46], will be assessed. For PLD, the approved regimen for standard use in the second-line management of ovarian cancer of $50 \mathrm{mg} / \mathrm{m}^{2}$ every 28 days results in a high incidence (20-30\%) of grade 3 'hand-foot syndrome' [47]. However, equivalent clinical activity with a substantially less severe adverse event profile has been observed when this agent is administered at $40 \mathrm{mg} / \mathrm{m}^{2}$ on a 4 week schedule [47]. Additional clinical evidence supports the conclusion that a $40 \mathrm{mg} / \mathrm{m}^{2}$ dose level of PLD is therapeutically equivalent to the higher (and more toxic) dose [48-50] and thus is being evaluated in the current trial. For relapsed ovarian cancer patients, approved topotecan dosing is $1.5 \mathrm{mg} / \mathrm{m}^{2}$ administered on days $1-5$ of a 3-week schedule. However, reduced dosing is widely used in standard clinical practice (e.g., $1.25 \mathrm{mg} / \mathrm{m}^{2}$ ), which confers decreased toxicity and similar outcome [51]. In addition, similar effectiveness with significantly lower hematologic toxicity has been observed in several studies when topotecan is administered on a weekly schedule [52]. Based on this evidence, a conventional $\left(1.25 \mathrm{mg} / \mathrm{m}^{2}\right.$ on days $1-5$ of a 3 -week schedule) and an alternative $\left(4.0 \mathrm{mg} / \mathrm{m}^{2}\right.$ on days 1,8 and 15 of a 4 -week cycle) schedule of topotecan administration were selected for FORWARD I. 


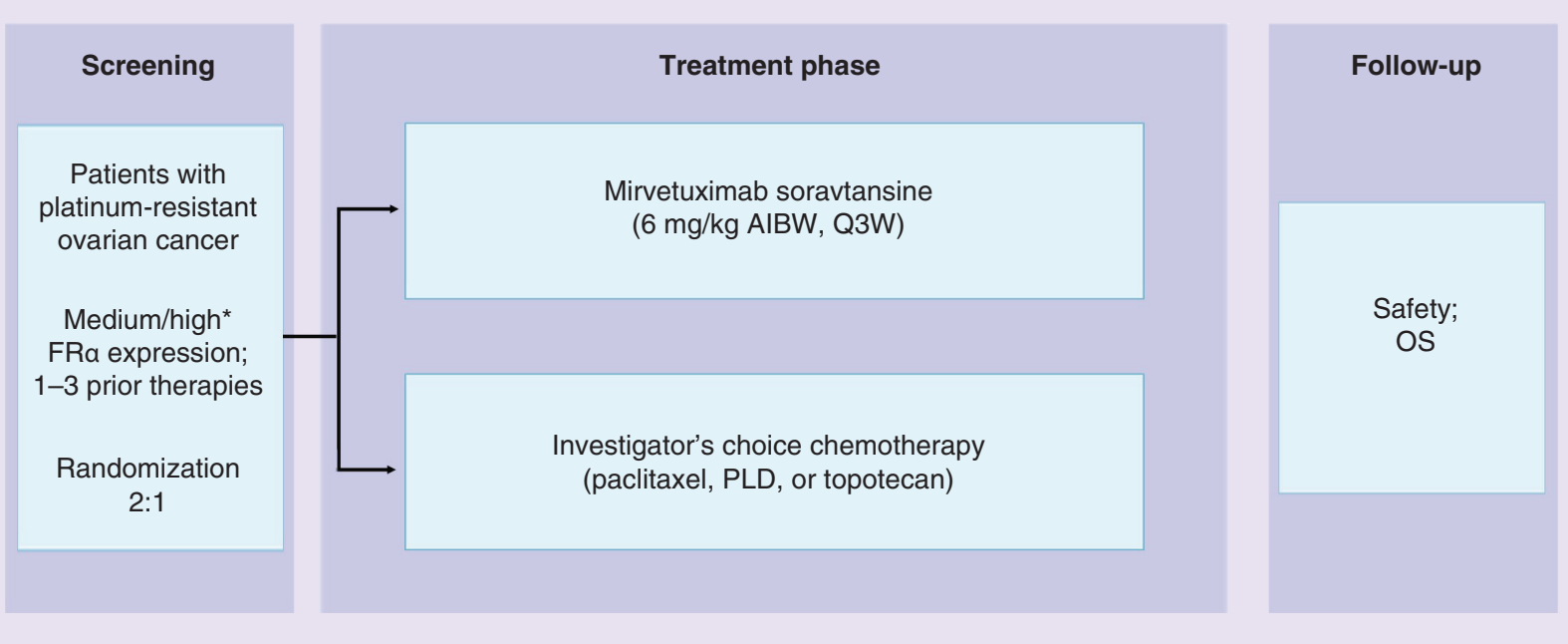

Figure 2. FORWARD I trial study design.

AIBW: Adjusted ideal body weight; OS: Overall survival; PLD: Pegylated liposomal doxorubicin.

\section{Design}

\section{Study design}

The study consists of a screening period, a treatment period and a follow-up period (Figure 2). During the screening phase, determination of patient eligibility, baseline characteristics, disease evaluation and clinical assessments are performed. Patients will be stratified by number of prior lines of therapy (1 or 2 vs 3), FR $\alpha$ levels (high, defined as $\geq 75 \%$ tumor staining at $\geq 2+$ intensity vs medium, defined as $\geq 50 \%$ and $<75 \%$ tumor staining at $\geq 2+$ intensity) and IC chemotherapy (paclitaxel vs PLD vs topotecan). Investigators will specify the chemotherapy of choice before randomization. Eligible patients will be randomized 2:1 into one of two arms as follows:

- Arm1: mirvetuximab soravtansine

- Arm 2: investigator's choice chemotherapy (paclitaxel, PLD or topotecan)

The treatment phase extends from randomization until disease progression (per Response Evaluation Criteria in Solid Tumors [RECIST] version 1.1 as assessed by the blinded independent review committee), development of unacceptable toxicity or withdrawal of consent. A safety follow-up visit will occur 30 days after the last treatment and all patients are followed every 3 months until death, lost to follow-up or withdrawal of consent for survival follow-up. Patients who withdraw or are removed from the study will have an end of treatment visit within 7 days of the decision to discontinue study treatment and those who discontinue study treatment for reasons other than progressive disease will continue tumor assessments every 12 weeks ( \pm 3 weeks) until documentation of progressive disease, withdrawal of consent or until the patient starts subsequent anticancer therapy, whichever comes first. For the purpose of evaluating PFS2 (time to second disease progression), information related to disease progression during survival follow-up and details regarding subsequent anticancer therapies will be collected. Data on the patient's functioning, health-related quality of life, disease symptoms and health status will be collected as part of the patient-reported outcomes.

\section{Primary \& secondary objectives}

The primary objective of the study is to compare the PFS of patients randomized to mirvetuximab soravtansine versus IC chemotherapy, as assessed by a blinded independent review committee, in both the intent to treat (ITT) population (i.e., all randomized patients) and in the high FR $\alpha$ subgroup ( $\geq 75 \%$ of tumor staining at $\geq 2+$ intensity). Key secondary objectives include the comparison of ORR, overall survival, duration of response and comparison of the primary patient-reported outcome end point in patients randomized to mirvetuximab soravtansine versus IC chemotherapy. End of study is defined as 1 year after the final analysis for the primary end point of PFS is conducted. 


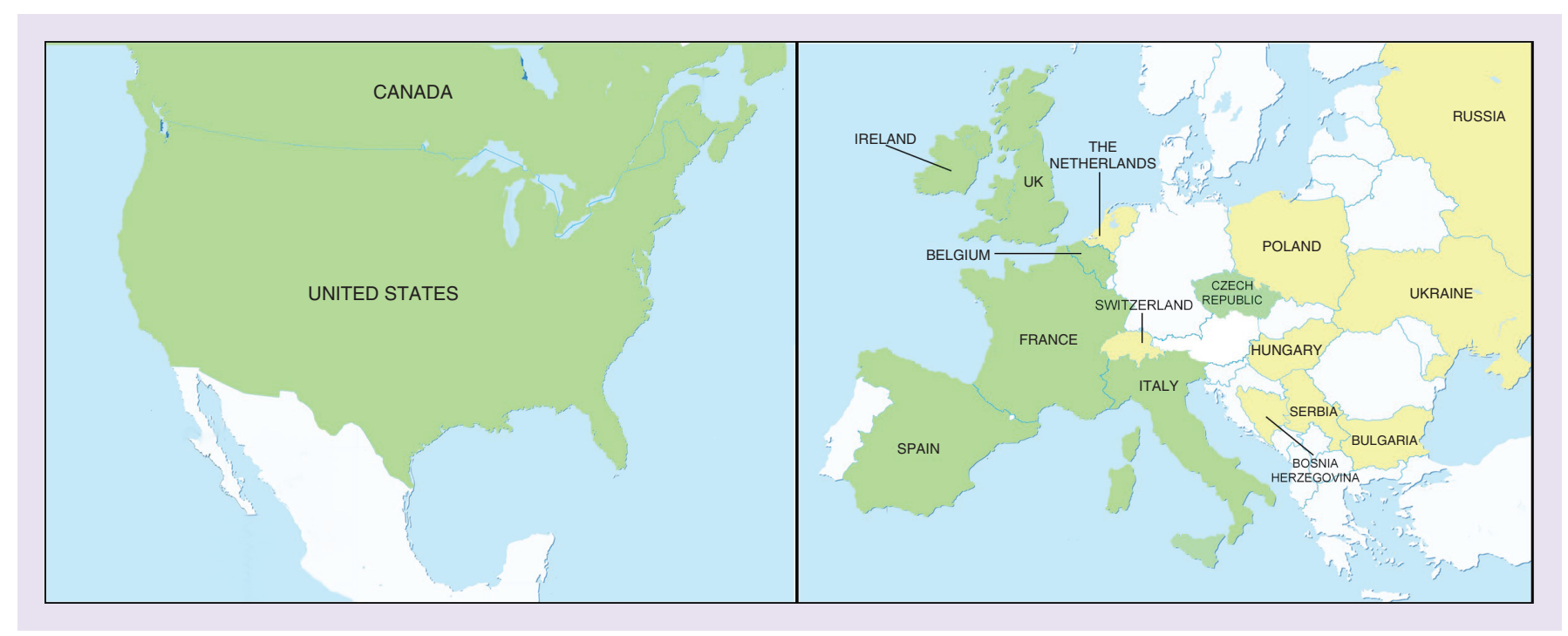

Figure 3. Participating countries in the FORWARD I trial. Countries with active sites are shown in green, those with pending sites are shown in yellow (as of January 2018).

\section{Key eligibility criteria}

Eligible patients are required to have a histologically confirmed diagnosis of EOC, primary peritoneal cancer or fallopian tube cancer; platinum-resistant disease, defined as progression within 6 months from completion of a minimum of four cycles of platinum-containing therapy; at least one lesion that meets the definition of measurable disease by RECIST v1.1; have received at least one, but no more than three, prior systemic lines of anticancer therapy; and meet the threshold of FR $\alpha$-positivity criteria on archival tumor samples (or, if unavailable, provide fresh biopsy) by validated immunohistochemistry-based assay at a central laboratory. Patients must also be at least 18 years of age, have an Eastern Cooperative Oncology Group performance status of 0 or 1, and have adequate hematologic, liver, cardiac and kidney function.

Patients meeting any of the following criteria are excluded: low-grade ovarian cancer or tumors of clear cell, mucinous or sarcomatous histology; primary platinum-refractory disease (defined by those individuals who progressed during or within 4 weeks of completion of their first platinum-based chemotherapy); greater than grade 1 peripheral neuropathy; active or chronic ocular disorders; history of neurological conditions or concurrent neurological condition that would confound assessment of treatment-emergent neuropathy; prior wide-field radiotherapy affecting at least $20 \%$ of the bone marrow; uncontrolled bleeding disorders or inadequate coagulation parameters; and history of hemorrhagic or ischemic stroke within the previous 6 months.

\section{Planned sample size}

FORWARD I is a multinational trial to be conducted at approximately 130 sites globally, with nine countries in North America and Europe, active and enrolling as of January 2018 (Figure 3). A total number of 333 patients are planned, who will be randomized 2:1 into the mirvetuximab soravtansine and chemotherapy arms (i.e., 222 and 111 patients, respectively). The primary end point of the study is PFS, in all randomized patients as well as in the FR $\alpha$ high expression subgroup. Sample size and power were determined by simulations using SAS software. The Hochberg procedure will be used to control the study-wise two-sided type I error. This design has a 91\% power to detect a hazard ratio of 0.583 in the FR $\alpha$ high expression subgroup and $96 \%$ power in all randomized patients at a study-wise $\alpha$ level of 5\%. An interim futility analysis will be conducted when at least 80 PFS events are observed and the final analysis is expected in the first half of 2019.

\section{Dose \& schedule of therapy}

Patients enrolled in arm 1 will receive mirvetuximab soravtansine at $6 \mathrm{mg} / \mathrm{kg}$ (AIBW) administered intravenously on day 1 of a 3-week cycle. Patients in arm 2 will receive paclitaxel, PLD or topotecan. Dosing and treatment schedules for all regimens are presented in Table 1. 


\begin{tabular}{|c|c|c|c|}
\hline Group & Drug & Dose & Dosing schedule \\
\hline Arm 1 & Mirvetuximab soravtansine & $6 \mathrm{mg} / \mathrm{kg}$ (AlBW) & Day 1 of a 3-week cycle \\
\hline \multirow[t]{4}{*}{ Arm 2} & Paclitaxel & $80 \mathrm{mg} / \mathrm{m}^{2}$ & Days $1,8,15$ and 22 of a 4-week cycle \\
\hline & PLD & $40 \mathrm{mg} / \mathrm{m}^{2}$ & Day 1 of a 4-week cycle \\
\hline & Topotecan (4-week cycle) & $4 \mathrm{mg} / \mathrm{m}^{2}$ & Days 1,8 and 15 of a 4-week cycle \\
\hline & Topotecan (3-week cycle) & $1.25 \mathrm{mg} / \mathrm{m}^{2}$ & Days $1-5$ of a 3-week cycle \\
\hline
\end{tabular}

\section{Efficacy evaluations}

Tumor response will be assessed using RECIST 1.1 and the blinded independent review committee (BIRC) assessment will be used for the primary end point (PFS) analysis. Radiographic tumor evaluation by computed tomography or MRI of chest, abdomen and pelvis is performed within 28 days prior to first dose of study treatment, every 6 weeks for the first 36 weeks on study and every 12 weeks thereafter. The same method of radiographic assessment used at screening is required at all subsequent radiographic evaluations. In addition to being reviewed by the investigator, radiographic scans are sent to a central imaging vendor, who is responsible for the formation and management of the blinded independent review committee. Serum CA-125 assessments will be performed within 14 days prior to the first treatment, at day 1 ( \pm 1 week) of each cycle, and at each radiologic tumor evaluation thereafter.

\section{Safety evaluations}

All individuals who receive treatment (i.e., at least one dose of study drug) will be considered evaluable for safety analyses. The safety in each treatment arm is assessed by evaluating adverse events, serious adverse events and deaths, as well as toxicities determined through changes in vital sign and clinical laboratory parameters. In addition, an ophthalmic examination will be performed at screening by an ophthalmologist, and ocular symptom assessment will be performed prior to the start of each cycle by the treating physician or other qualified individual. For patients reporting greater than grade 1 ocular symptoms, treatment will be held until the patient is re-evaluated by an ophthalmologist for a complete examination.

\section{Statistical analyses}

Demographics and baseline characteristics will be summarized using descriptive statistics. For categorical variables, the number (n) and percent of each category within a parameter will be presented. For continuous variables, the sample size (n), mean, median and standard deviation, as well as the minimum and maximum values, will be determined. Efficacy analyses will be performed on the ITT population, defined as all randomized patients. The primary end point of PFS will be estimated using Kaplan-Meier method and comparison between treatment arms conducted using Cox proportional hazard regression and log-rank test, stratified by FR $\alpha$ expression level (for ITT analysis only, high vs medium), IC chemotherapy (paclitaxel vs PLD vs topotecan) and number of prior lines of therapy ( 1 or 2 vs 3 ). The secondary end point of ORR, defined as the proportion of patients with a confirmed complete response or partial response per RECIST 1.1, will be estimated along with a 95\% CI; additional secondary end points of overall survival, duration of response and PFS (as assessed by the investigator) will be estimated using Kaplan-Meier method. Comparison between treatment arms will be conducted using Cox proportional hazard regression and log-rank tests.

\section{Conclusion}

The FORWARD I trial described here will investigate the efficacy and tolerability of mirvetuximab soravtansine versus IC chemotherapy in patients with advanced, platinum-resistant ovarian cancer. The results of this study will help define the role of this novel targeted agent in the management of relapsed/refractory ovarian cancer, with a clear potential to improve outcomes in this difficult-to-treat patient population. 
Executive summary

\section{Background}

- Ovarian cancer remains a leading cause of gynecological cancer mortality worldwide. Standard-of-care treatment, surgery followed by adjuvant platinum-based chemotherapy, has largely reached a plateau of effectiveness in improving overall patient survival.

- The development of platinum-resistant disease imparts a particularly dismal prognosis for patients; new, effective therapeutic options represent an urgent unmet medical need for this population.

- Elevated folate receptor- $\alpha(F R \alpha)$ expression is characteristic of epithelial ovarian cancer (EOC), and the differential distribution pattern of this receptor has been investigated as a means to selectively target tumors in order to maximize antitumor efficacy and treatment tolerability.

Mirvetuximab soravtansine

- Mirvetuximab soravtansine is the first FR $\alpha$-targeting antibody-drug conjugate to enter human trials, and comprises a cytotoxic effector compound, the maytansinoid DM4, coupled to a humanized anti-FR $\alpha$ monoclonal antibody.

- As an antibody-drug conjugate, mirvetuximab soravtansine couples the targeting and pharmacokinetic features of its antibody moiety with the additional cancer-killing impact of the cytotoxic payload.

- The Phase I clinical experience with mirvetuximab soravtansine demonstrated manageable tolerability and encouraging signals of activity, particularly in patients with platinum-resistant EOC.

FORWARD I study

- An open label, randomized Phase III trial to compare the safety and efficacy of mirvetuximab soravtansine versus investigator's choice of chemotherapy in women with FR $\alpha$-positive, platinum-resistant EOC, primary peritoneal cancer or fallopian tube cancer.

- Retrospective and expansion cohort analyses from the early-phase clinical evaluations demonstrated that platinum-resistant patients with one to three prior lines of therapy and medium/high FR $\alpha$ expression exhibited the strongest signals of efficacy, thus identifying the target population for this study.

\section{Conclusion}

- The results of this pivotal monotherapy trial will help define the role of this novel targeted agent for improving outcomes in this difficult to treat patient population.

Supplementary data

An infographic accompanies this paper. To download the infographic that accompanies this paper, please visit the journal website at: www.futuremedicine.com/doi/10.2217/fon-2017-0646

Acknowledgements

The authors would like to thank Richard Bates Sr, Manager Publications at ImmunoGen, Inc. for drafts and editorial assistance during the production of this article.

Financial \& competing interests disclosure

K Malek is an employee of ImmunoGen, Inc. KN Moore and MJ Birrer serve on an advisory board for ImmunoGen. The authors have no other relevant affiliations or financial involvement with any organization or entity with a financial interest in or financial conflict with the subject matter or materials discussed in the manuscript apart from those disclosed.

No writing assistance was utilized in the production of this manuscript.

Open access

This work is licensed under the Attribution-NonCommercial-NoDerivatives 4.0 Unported License. To view a copy of this license, visit http://creativecommons.org/licenses/by-nc-nd/4.0/

\section{References}

Papers of special note have been highlighted as: $\bullet$ of interest; $\bullet \bullet$ of considerable interest

1. Ferlay J, Soerjomataram I, Dikshit $\mathrm{R}$ et al. Cancer incidence and mortality worldwide: sources, methods and major patterns in GLOBOCAN 2012. Int. J. Cancer 136(5), e359-e386 (2015).

2. Reid BM, Permuth JB, Sellers TA. Epidemiology of ovarian cancer: a review. Cancer Biol. Med. 14(1), 9-32 (2017).

3. Lacey JV, Sherman ME. Ovarian neoplasia. In: Robboy's Pathology of the Female Reproductive Tract. Robboy SL, Mutter GL, Prat J (Eds). Churchill Livingstone, Elsevier, Oxford, UK, 601 (2009).

4. Desai A, Xu J, Aysola K et al. Epithelial ovarian cancer: an overview. World J. Transl. Med. 3(1), 1-8 (2014). 
5. Davis A, Tinker AV, Friedlander M. 'Platinum resistant' ovarian cancer: what is it, who to treat and how to measure benefit? Gynecol. Oncol. 133(3), 624-631 (2014).

6. Markman M, Bookman MA. Second-line treatment of ovarian cancer. Oncologist 5(1), 26-35 (2000).

7. Luvero D, Milani A, Ledermann JA. Treatment options in recurrent ovarian cancer: latest evidence and clinical potential. Ther. Adv. Med. Oncol. 6(5), 229-239 (2014).

8. Pujade-Lauraine E, Hilpert F, Weber B et al. Bevacizumab combined with chemotherapy for platinum-resistant recurrent ovarian cancer: the AURELIA open-label randomized Phase III trial. J. Clin. Oncol. 32(13), 1302-1308 (2014).

-• Pivotal Phase III trial results that resulted in approval of bevacizumab for the treatment of ovarian cancer.

9. Poveda AM, Selle F, Hilpert F et al. Bevacizumab combined with weekly paclitaxel, pegylated liposomal doxorubicin, or topotecan in platinum-resistant recurrent ovarian cancer: analysis by chemotherapy cohort of the randomized Phase III AURELIA trial. J. Clin. Oncol. 33(32), 3836-3838 (2015).

10. Pistollato F, Calderon Iglesias R, Ruiz $\mathrm{R}$ et al. The use of natural compounds for the targeting and chemoprevention of ovarian cancer. Cancer Lett. 411, 191-200 (2017).

11. Liu J, Matulonis UA. New strategies in ovarian cancer: translating the molecular complexity of ovarian cancer into treatment advances. Clin. Cancer Res. 20(20), 5150-5156 (2014).

12. Della Pepa C, Tonini G, Pisano C et al. Ovarian cancer standard of care: are there real alternatives? Chin. J. Cancer 34(1), 17-27 (2015).

13. Colombo N, Conte PF, Pignata S, Raspagliesi F, Scambia G. Bevacizumab in ovarian cancer: focus on clinical data and future perspectives. Crit. Rev. Oncol. Hematol. 97, 335-348 (2016).

14. Konecny GE, Kristeleit RS. PARP inhibitors for BRCA1/2-mutated and sporadic ovarian cancer: current practice and future directions. Br. J. Cancer 115(10), 1157-1173 (2016).

15. Vergote IB, Marth C, Coleman RL. Role of the folate receptor in ovarian cancer treatment: evidence, mechanism, and clinical implications. Cancer Metastasis Rev. 34(1), 41-52 (2015).

- Summarizes role of folate receptor in ovarian cancer, providing a rationale for targeting this receptor in the development of new therapeutic agents.

16. Choi SW, Mason JB. Folate and carcinogenesis: an integrated scheme. J. Nutr. 130(2), 129-132 (2000).

17. Kalli KR, Oberg AL, Keeney GL et al. Folate receptor $\alpha$ as a tumor target in epithelial ovarian cancer. Gynecol. Oncol. 108(3), 619-626 (2008).

18. Markert S, Lassmann S, Gabriel B et al. Alpha-folate receptor expression in epithelial ovarian carcinoma and non-neoplastic ovarian tissue. Anticancer Res. 28(6A), 3567-3572 (2008).

19. O'Shannessy DJ, Somers EB, Smale R, Fu YS. Expression of folate receptor- $\alpha$ (FRA) in gynecologic malignancies and its relationship to the tumor type. Int. J. Gynecol. Pathol. 32(3), 258-268 (2013).

20. Toffoli G, Russo A, Gallo A et al. Expression of folate binding protein as a prognostic factor for response to platinum-containing chemotherapy and survival in human ovarian cancer. Int. J. Cancer 79(2), 121-126 (1998).

21. Marchetti C, Palaia I, Giorgini M et al. Targeted drug delivery via folate receptors in recurrent ovarian cancer: a review. Onco Targets Ther. 7, 1223-1236 (2014).

22. Assaraf YG, Leamon CP, Reddy JA. The folate receptor as a rational therapeutic target for personalized cancer treatment. Drug Resist. Updat. 17(4-6), 89-95 (2014).

23. Ledermann JA, Canevari S, Thigpen T. Targeting the folate receptor: diagnostic and therapeutic approaches to personalize cancer treatments. Ann. Oncol. 26(10), 2034-2043 (2015).

24. Vergote I, Armstrong D, Scambia G et al. A randomized, double-blind, placebo-controlled, Phase III study to assess efficacy and safety of weekly farletuzumab in combination with carboplatin and taxane in patients with ovarian cancer in first platinum-sensitive relapse. $J$. Clin. Oncol. 34(19), 2271-2278 (2016).

25. Levin J. Merck and endocyte announce independent DSMB recommends vintafolide PROCEED Phase III trial be stopped for futility following interim analysis. FierceBiotech (2014). www.fiercebiotech.com/biotech/merck-and-endocyte-announce-independent-dsmb-re commends-vintafolide-proceed-phase-3-trial

26. Chari RV, Miller ML, Widdison WC. Antibody-drug conjugates: an emerging concept in cancer therapy. Angew. Chem. Int. Ed. Engl. 53(15), 3796-3827 (2014).

27. Parslow AC, Parakh S, Lee FT, Gan HK, Scott AM. Antibody-drug conjugates for cancer therapy. Biomedicines 4(3), e254-e262 (2016).

28. Thomas A, Teicher BA, Hassan R. Antibody-drug conjugates for cancer therapy. Lancet Oncol. 17(6), e254-e262 (2016).

29. Lambert JM, Morris CQ. Antibody-drug conjugates (ADCs) for personalized treatment of solid tumors: a review. Adv. Ther. 34(5), 1015-1035 (2017).

30. Lambert JM, Berkenblit A. Antibody-drug conjugates for cancer treatment. Annu. Rev. Med. 17(6), e254-e262 (2017).

- Comprehensive review of the current status of antibody-drug conjugates undergoing advanced clinical testing. 
31. Gunderson CC, Moore KM. Mirvetuximab soravtansine. Folate receptor $\alpha$ (FR- $\alpha)$-targeting antibody drug conjugate, treatment of epithelial ovarian cancer. Neoplasia 18(12), 775-784 (2016).

32. Oroudjev E, Lopus M, Wilson L et al. Maytansinoid-antibody conjugates induce mitotic arrest by suppressing microtubule dynamic instability. Mol. Cancer Ther. 9(10), 2700-2713 (2010).

33. Goldmacher VS, Audette CA, Guan Y et al. High-affinity accumulation of a maytansinoid in cells via weak tubulin interaction. PLoS ONE 10(2), e0117523 (2015).

34. Kovtun YV, Audette CA, Ye Y et al. Antibody-drug conjugates designed to eradicate tumors with homogeneous and heterogeneous expression of the target antigen. Cancer Res. 66(6), 3214-3221 (2006).

35. Ab O, Whiteman KR, Bartle LM et al. IMGN853, a folate receptor- $\alpha$ (FR- $\alpha$ )-targeting antibody-drug conjugate, exhibits potent targeted antitumor activity against FR- $\alpha$-expressing tumors. Mol. Cancer Ther. 14(7), 1605-1613 (2015).

- Preclinical development and characterization of mirvetuximab soravtansine.

36. Ponte JF, Ab O, Lanieri L et al. Mirvetuximab soravtansine (IMGN853), a folate receptor $\alpha$-targeting antibody-drug conjugate, potentiates the activity of standard of care therapeutics in ovarian cancer models. Neoplasia 18(12), 775-784 (2016).

37. Moore KN, Borghaei H, O'Malley DM et al. Phase I dose-escalation study of mirvetuximab soravtansine (IMGN853), a folate receptor $\alpha$-targeting antibody-drug conjugate, in patients with solid tumors. Cancer 123(16), 3080-3087 (2017).

38. Eaton JS, Miller PE, Mannis MJ, Murphy CJ. Ocular adverse events associated with antibody-drug conjugates in human clinical trials. J. Ocul. Pharmacol. Ther. 31(10), 589-604 (2015).

39. Martin LP, Konner JA, Moore KN et al. Characterization of folate receptor $\alpha$ (FR- $\alpha$ ) expression in archival tumor and biopsy samples from relapsed epithelial ovarian cancer patients: a Phase I expansion study of the FR- $\alpha$-targeting antibody-drug conjugate mirvetuximab soravtansine. Gynecol. Oncol. 147(2), 402-407 (2017).

40. Moore KN, Martin LP, O’Malley DM et al. Safety and activity of mirvetuximab soravtansine (IMGN853), a folate receptor $\alpha$-targeting antibody-drug conjugate, in platinum-resistant ovarian, fallopian tube, or primary peritoneal cancer: a Phase I expansion study. J. Clin. Oncol. 35(10), 1112-1118 (2017).

-. Findings of a Phase I expansion cohort study indicating that mirvetuximab soravtansine is clinically active in patients with platinum-resistant epithelial ovarian cancer.

41. Moore KN, Matulonis UA, O’Malley DM et al. Mirvetuximab soravtansine (IMGN853), a folate alpha (FR- $\alpha$ )-targeting antibody-drug conjugate (ADC), in platinum-resistant epithelial ovarian caner (EOC) patients (pts): activity and safety analyses in Phase I pooled expansion cohorts. J. Clin. Oncol. 35(Suppl.), Abstract 5547 (2017).

42. Marupudi NI, Han JE, Li KW, Renard VM, Tyler BM, Brem H. Paclitaxel: a review of adverse toxicities and novel delivery strategies. Expert Opin. Drug Saf. 6(5), 609-621 (2007).

43. Rosenberg $\mathrm{P}$, Andersson $\mathrm{H}$, Boman $\mathrm{K}$ et al. Randomized trial of single agent paclitaxel given weekly versus every three weeks and with peroral versus intravenous steroid premedication to patients with ovarian cancer previously treated with platinum. Acta Oncol. 41(5), 418-424 (2002).

44. Markman M, Hall J, Spitz D et al. Phase II trial of weekly single-agent paclitaxel in platinum/paclitaxel-refractory ovarian cancer. J. Clin. Oncol. 20(9), 2365-2369 (2002).

45. Gynecologic Oncology Group, Markman M, Blessing J et al. Phase II trial of weekly paclitaxel $\left(80 \mathrm{mg} / \mathrm{m}^{2}\right)$ in platinum and paclitaxel-resistant ovarian and primary peritoneal cancers: a Gynecologic Oncology Group study. Gynecol. Oncol. 101(3), 436-440 (2006).

46. Baird RD, Tan DS, Kaye SB. Weekly paclitaxel in the treatment of recurrent ovarian cancer. Nat. Rev. Clin. Oncol. 7(10), 575-582 (2010).

47. Markman M. Pegylated liposomal doxorubicin: appraisal of its current role in the management of epithelial ovarian cancer. Cancer Manag. Res. 3, 219-225 (2011).

48. Markman M, Kennedy A, Webster K, Peterson G, Kulp B, Belinson J. Phase II trial of liposomal doxorubicin $\left(40 \mathrm{mg} / \mathrm{m}\left({ }^{2}\right)\right)$ in platinum/paclitaxel-refractory ovarian and fallopian tube cancers and primary carcinoma of the peritoneum. Gynecol. Oncol. 78(3 Pt 1), 369-372 (2000).

49. Wilailak S, Linasmita V. A study of pegylated liposomal doxorubicin in platinum-refractory epithelial ovarian cancer. Oncology 67(3-4), 183-186 (2004).

50. Nakayama M, Kobayashi H, Takahara T, Nishimura Y, Fukushima K, Yoshizawa K. A comparison of overall survival with 40 and $50 \mathrm{mg} / \mathrm{m}^{2}$ pegylated liposomal doxorubicin treatment in patients with recurrent epithelial ovarian cancer: propensity score-matched analysis of real-world data. Gynecol. Oncol. 143(2), 246-251 (2016).

51. Sehouli J, Oskay-Ozcelik G. Current role and future aspects of topotecan in relapsed ovarian cancer. Curr. Med. Res. Opin. 25(3), 639-651 (2009).

52. Sehouli J, Stengel D, Harter P et al. Topotecan weekly versus conventional 5-day schedule in patients with platinum-resistant ovarian cancer: a randomized multicenter Phase II trial of the North-Eastern German Society of Gynecological Oncology Ovarian Cancer Study Group. J. Clin. Oncol. 29(2), 242-248 (2011). 